\title{
Bone Texture Characterization Based on Contourlet and Gabor Tranforms
}

\author{
Viet Quoc Ngo and Thuc Nguyen Dinh
}

\begin{abstract}
In this paper, we proposed the new texture features called Haralick in Contourlet Sub-bands based FeatureHCSF-for 2D bone structure evaluation. Our proposed bone texture features are built on co-occurrences matrix for outside wedges and Gabor features for coarsest level of contourlet transform. The classification experiments are tested with linear SVM engine and the precision ratio is $70 \%$. The proposed method is also compared to SFTA algorithm, Haralick, and Gabor features. The classification precision of proposed method is always higher than those in the related methods.
\end{abstract}

Index Terms-Bone texture characterization, contourlet, gabor transform.

\section{INTRODUCTION}

Texture Characterization of Bone radiograph images is a challenge in the osteoporosis diagnosis. Osteoporosis is defined as a skeletal disorder characterized by compromised bone strength predisposing to an increased risk of fracture [1]. The most common method for osteoporosis diagnosis is to estimate Bone Mineral Density (BMD) by dual-energy Xray absorptiometry [2]. However, BMD alone represents only $60 \%$ of fracture prediction. The characterization of trabecular bone microarchitecture has been recognized as an important factor and completes the osteoporosis diagnosis using BMD [3], but it cannot be routinely obtained by noninvasive methods and requires a bone biopsy with histomorphometric analysis. 2D texture analysis offers a simple way to evaluate bone structure on conventional radiographs. The evaluation of osteoporotic disease from bone radiograph images presents a major challenge for pattern recognition and medical applications. Textured images from the bone microarchitecture of osteoporotic and healthy subjects show a high degree of similarity, thus drastically increasing the difficulty of classifying such textures. Fig. 1 shows the bone texture similarities of control and osteoporotic images.

In order to deal with classification qualities, we propose new features for these bone textures. The features are extracted from the novel combination of contourlet transform, co-occurrence matrix, and Gabor filter banks.

We evaluated the proposed features on bone dataset from "Challenge IEEE-ISBI: Bone Texture Characterization" with Linear SVM.

Manuscript received September 28, 2014; revised February 2, 2015.

Viet Quoc Ngo is with University of Education, HCMC, Vietnam (email: thanhbinhsp@gmail.com).

Thuc Nguyen Dinh is with Hochiminh City University of Natural Science, HCMC, Vietnam (e-mail: ndthuc@fit.hcmus.edu.vn).
We also compared proposed features to widely employed texture extraction methods such as Haralick descriptors [4], Gabor filter banks and SFTA algorithm [5]. In the experiments, the proposed feature has shown more precision than those in the related works.

The paper is organized as follows. Section II summaries the result of related works in texture analysis. Section III details our proposed method. Section IV presents the experiments classification on bone dataset and comparisons to other methods. Finally, section Vis for conclusion.
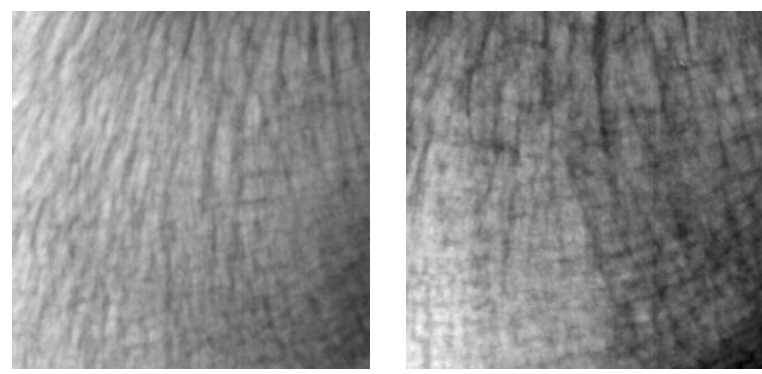

Fig. 1. The similarity of control and osteoporotic images.

\section{RELATED WORKS}

2D Texture plays important role in pattern recognition. Textures can be considered as patterns in which the statistics such as mean, standard deviation, entropy, and others can be used for characterization. There are many achievements in this field, such as Haralick, GLCM [6] features, Gabor filter.

Gabor filter is a popular of filter bank-based method. Each Gabor filter is represented as a Gaussian function. The following equation shows the formal representation of a Gabor filter.

$$
g(x, y, \lambda, \theta, \sigma)=\frac{1}{2 \pi \sigma^{2}} \exp \left(-\frac{1}{2} A+\frac{2 \pi i R_{1}}{\lambda}\right)
$$

where

$$
A=\left(\frac{R_{1}^{2}+R_{2}^{2}}{\sigma^{2}}\right),\left[\begin{array}{l}
R_{1} \\
R_{2}
\end{array}\right]=\left[\begin{array}{cc}
\cos \theta & \sin \theta \\
-\sin \theta & \cos \theta
\end{array}\right]\left[\begin{array}{l}
x \\
y
\end{array}\right], \quad \theta \text { is a filter }
$$

orientation, $\sigma$ is standard deviation, $\lambda$ is wavelength of the sinusoid, and $x \in[1, m], y \in[1, n]$, with $m x n$ is filter size. Gabor filter banks are popular for texture classification. Frequency and orientation representations of the Gabor filter are similar to those of the human visual system. A set of Gabor filters with different frequencies and orientations may be helpful for extracting useful features from an image. The images are filtered using the real parts of various different Gabor filter kernels. The mean and variance of the filtered images are used as features for classification.

Fig. 2 shows that the filtered images of two distinguish classes via Gabor filter banks are too similar. The 
experiments using Gabor filters for bone texture show that these features are not suitable for undertaken problem.

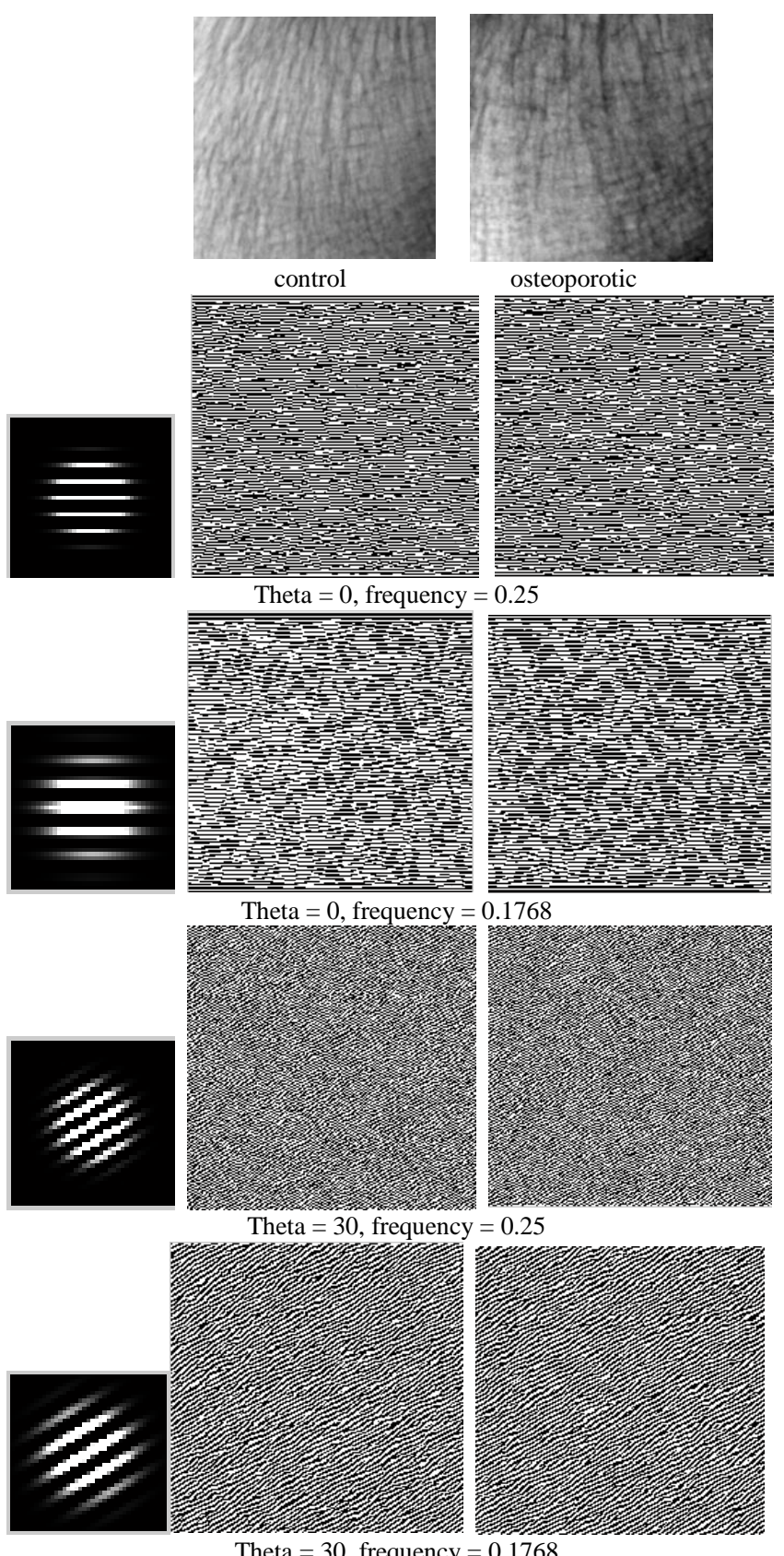

Theta $=30$, frequency $=0.1768$

Fig. 2. Filtered images via gabor filters.

Recently, the authors in [5] proposed the SFTA algorithm for texture classification. The algorithm can be divided to two parts: input gray scale image is segmented to set of binary images based on Otsu method; fractal features are extracted for every binary image. More particular, the TwoThreshold Binary Decomposition (TTBD) estimates a set $\mathrm{T}$ of thresholds automatically which is based on multi-level Otsu algorithm. From these estimated thresholds called $\left\{t_{u}\right\}$, decomposition step in gray scale image $I(x, y)$ is executed to generate a set of binary images through twothreshold method.

$$
I_{b}(x, y)=\left\{\begin{array}{cc}
1 & t_{l}<I(x, y) \leq t_{u} \\
0 & \text { otherwise }
\end{array}\right.
$$

where $t_{l}$ and $t_{u}$ are adjacent lower and upper thresholds.
For each generated binary image, the SFTA feature vector is constructed with 3 components: size of binary image (number of foreground pixels), mean gray level, and boundaries' fractal dimension (using Box Count Algorithm).

The binary images segmented from SFTA algorithm for control and osteoporotic (from Fig. 1) also show the high similarities, so the features such as fractal dimension, mean gray level, and size of binary cannot be distinguished.

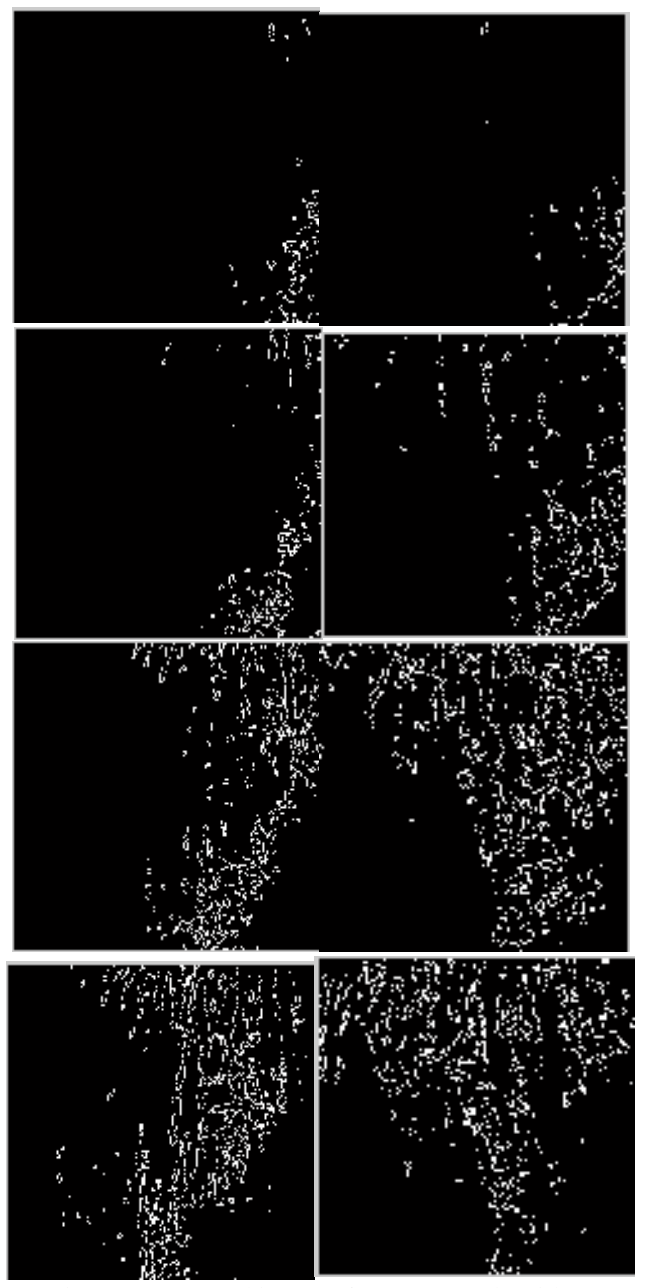

Fig. 3. Segmented images from SFTA algorithm.

The experiments show that these methods achieved high precision classification in datasets of distinguish textures such as KTH-TIPS, Texture Surfaces, Lung CT ROIs. Unfortunately, these methods do not classify well in bone texture datasets. The main reason is that most bone textures for healthy and osteoporotic images are too similarities. Via our experiments, highest precision for this dataset is Gabor filters and it only achieved $67 \%$ with linear SVM.

\section{PROPOSED METHOD}

The proposed method is divided to two main steps: image decomposition to generate a set of wedges via contourlet transforms; feature vector is constructed for each wedge consisting Gabor filter and Cluster Prominence.

\section{A. Contourlet Transform}

The contourlet transform [7] is implemented via twodimensional filter bank that decomposes an image to several directional sub-bands at multiple scales. The resulting image 
expansion is a directional multi-resolution sub-bands. It is built by combining the Laplace Pyramid with Directional Filter Bank (PDFB) at each scale. At each scale, we can decompose into any arbitrary power of two's number. Multiscale decomposition is done by a Laplacian pyramid, and then a directional decomposition is done using directional filter bank. This transform can be suited for several applications involving curve detection, as well as texture representation.

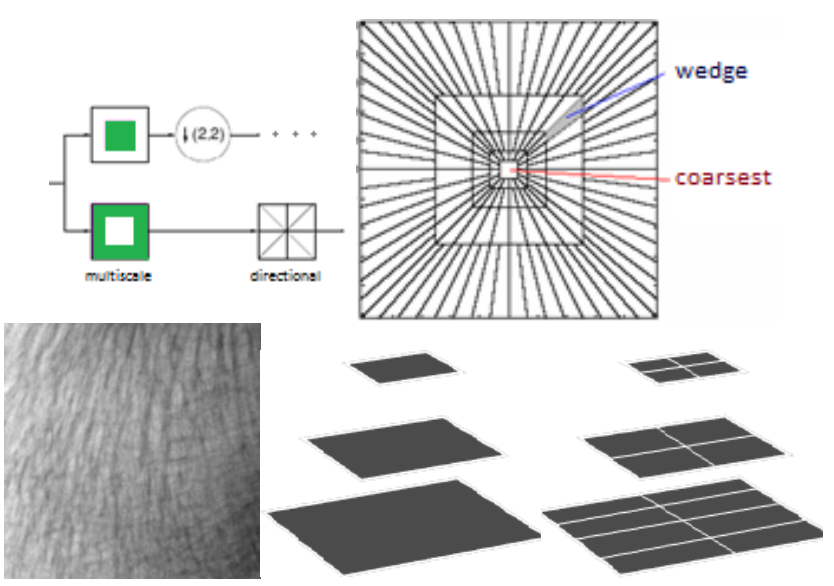

Fig. 4. The contourlet transform's diagrams.
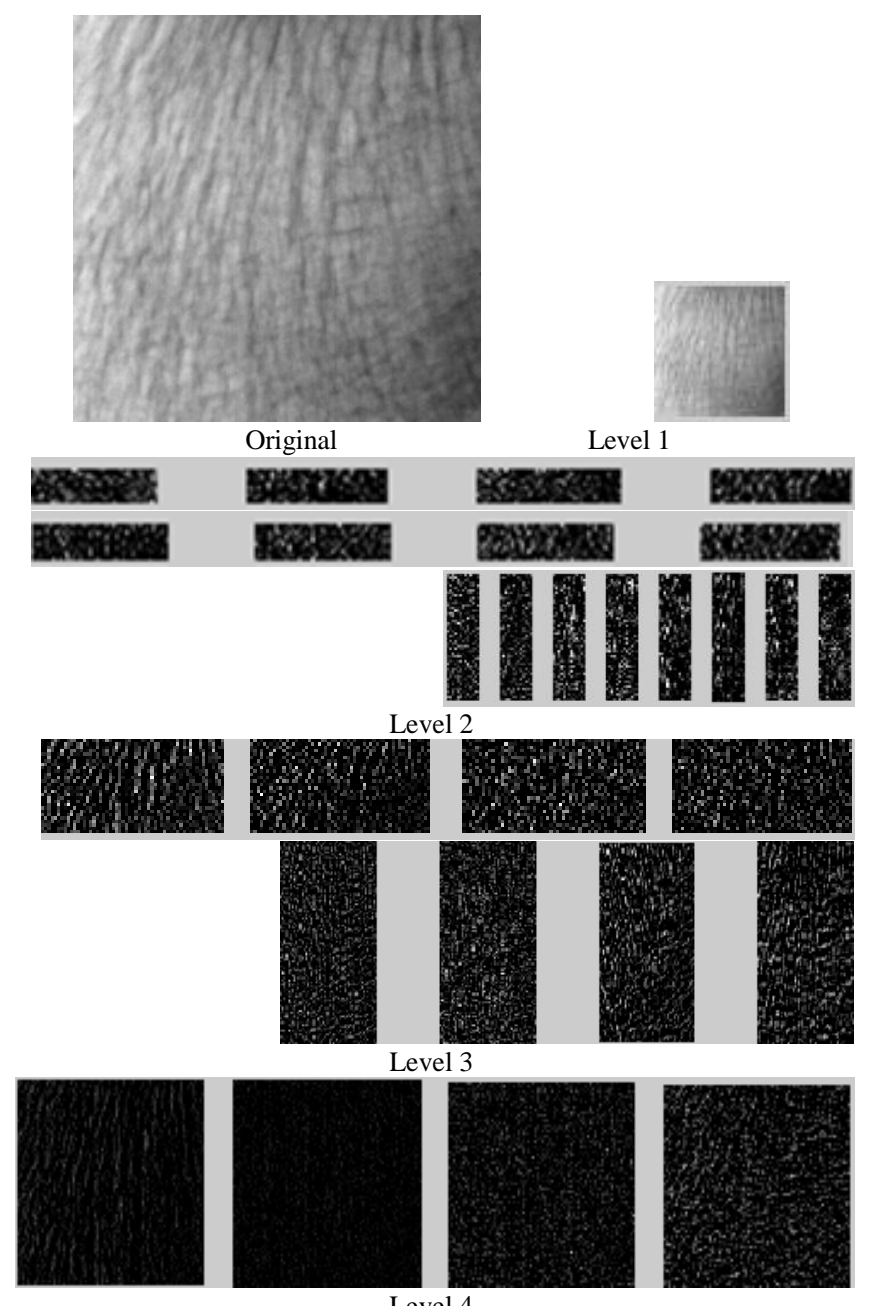

Level 4

Fig. 5. The bone texture image and its sub-bands generated from contourlet transform.

Via this transform, the image is partitioned to many patches called wedges in different scales and orientations.
Each wedge is determined by the orientations, and the scales. In order to build feature vector for image, we try to extract features for each wedge separately, and combine them to have complete vector. An amount of wedges depends on a number of scales and orientations. For each wedge, we use from 2 to 8 features at most. So the average feature vector length of an image is from 32 to 1024 (depended on a number of orientations for each scale).

Instead of using local features such as SIFT, SURF or global features such as HOG, we use pattern features for the patch. For SIFT or SURF, the key point detectors are hard to be suitable for the pattern or texture image patches. Meanwhile, Haralick features are proved to be good for pattern or texture classification.

The contourlet coefficients in each wedge (directional sub-band) can represent the prominence curves. Logically, we can keep the significant coefficients to keep the most prominence curve. However, one curve cannot represent all curves in the wedge. Therefore, in this case prominence cluster generated from GLCM matrix are best suitable for the set of curves in the wedge.

Fig. 5 shows an example of the contourlet transform on bone texture image $(400 \times 400)$. The image is decomposed into 4 scales with one low-pass sub-band (coarsest subband), and three band-pass directional sub-bands. This

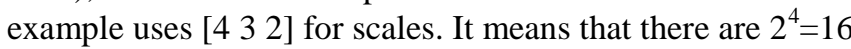
sub-bands $(13 \times 50)$ for the coarsest scale (level 2$), 2^{3}=8$ subbands $(50 \times 100)$ for the second finer scale (level 3$)$, and $2^{2}=4$ direction sub-bands $(200 \times 200)$ for last scale (level 4$)$. Besides, there is one sub-band $(50 \times 50)$ for the coarsest scale (level 1).

\section{B. Cluster Prominence Feature Vector}

For the low-pass sub-band, we apply Gabor transform. Meanwhile, for each directional sub-bands of any decomposition level, we use the cluster prominence of the gray level co-occurrence matrix.

Here is brief of GLCM and the Cluster Prominence feature that we are using for the directional sub-bands

Given an image with $G$ gray levels, GLCM is a matrix with size of $G x G$. The matrix element is determined by $P(i, j, \Delta x, \Delta y)$. It is the relative frequency with which two pixels separated by an offset $(\Delta x, \Delta y)$ occur within a given neighborhood, one with intensity $i$, and other with intensity $j$.

Given an $M x N$ neighborhood for an image with $G$ gray levels, let $f(m, n)$ be the intensity at the location $(m, n)$, then

$$
P(i, j, \Delta x, \Delta y)=W \cdot Q(i, j, \Delta x, \Delta y)
$$

where

$$
\begin{gathered}
W=\frac{1}{(M-\Delta x)(N-\Delta y)} \\
Q(i, j, \Delta x, \Delta y)=\sum_{n=1}^{N-\Delta y} \sum_{m=1}^{M-\Delta x} A
\end{gathered}
$$

$$
A= \begin{cases}1, & f(m, n)=i \text { and } f(m+\Delta x, n+\Delta y)=j \\ 0, & \text { else }\end{cases}
$$




$$
\begin{gathered}
\mu_{x}=\sum_{i=0}^{G-1} i \sum_{j=0}^{G-1} P(i, j)=\sum_{i=0}^{G-1} i P_{x}(i) \\
\mu_{y}=\sum_{i=0}^{G-1} \sum_{j=0}^{G-1} j P(i, j)=\sum_{i=0}^{G-1} j P_{y}(j) \\
P_{x}(i)=\sum_{j=0}^{G-1} P(i, j) \\
P_{y}(j)=\sum_{i=0}^{G-1} P(i, j)
\end{gathered}
$$

This Cluster Prominence is determined by

$$
\sum_{i=0}^{G-1} \sum_{j=0}^{G-1}\left(i+j-\mu_{x}-\mu_{y}\right)^{4} \times P(i, j)
$$

The coarsest sub-band in contourlet transform is square matrix. For 3-scale contourlet decomposition, its size is $50 \times 50$. Through many experiments of choosing the suitable features for this patch, we observe that Gabor filter with 4 main directions gives the little higher performance than 3 kinds of feature such as: GLCM matrix; the most significant values in the transform; or the popular features used in pattern recognition/classification.

\section{The proposed main features are as follows:}

- Sixteen values of Gabor transform for low-pass subband $(50 \times 50)$. We use Gabor filter $3 \times 3,2$ directions for each scale, and 4 scales. There are 16 coefficients for this sub-band.

- Two Cluster Prominence coefficients of each directional sub-bands. We use two pairs $[0,1]$, and $[1,0]$ for $(\Delta x, \Delta y)$. For the scales [ [ 432 ] $]$ as in Fig. 4, there are 56 coefficients of directional sub-bands.

\section{EXPERIMENTS}

Experiments are evaluated with dataset of 58 control, 58 osteoporotic and 58 blind images retrieved from worldwide challenge IEEE-ISBI 2014: http://www.univorleans.fr/i3mto/challenge-ieee-isbi-bone-texture-

characterization. The related works reviewed in this paper are very popular in pattern recognition, and texture characterization sessions. We try to retrieve original the resources (source code, dataset) from these works and execute the experiments in the same context. For each method, we have tested with a lot of various parameter sets. These parameters are depended on a number of thresholds, number of orientations (in Gabor and directional filter bank).

The scale sets of contourlet are tested and compared the results. We execute the classifications with Linear SVM engine, with $k_{\text {fold }} \in[3,11]$, termination criterion $=0.01$, $C \in[0.1,0.2, \cdots, 1.0]$. Every method is tested with combination of the parameters in SVM classifier. The proposed method is compared to that of the following methods: Haralick, Gabor, and SFTA. Table I shows feature

\begin{tabular}{|c|c|c|}
\hline Name & Description & Vector length \\
\hline SFTA & 8 thresholds & 48 \\
\hline Gabor & $\begin{array}{l}3 \text { scales, } 20 \text { orientations, down- } \\
\text { sampling } 4\end{array}$ & 240 \\
\hline GLCM & $\begin{array}{l}\text { All statistic features of GLCM, } 2 \\
\text { offsets }(\Delta x, \Delta y)\end{array}$ & 88 \\
\hline HSCF & $\begin{array}{l}16 \text { Gabor coefficients for low-pass }+56 \\
\text { ones for directional sub-bands }\end{array}$ & 72 \\
\hline
\end{tabular}

length in the experiments. We calculate the average results of each algorithm/method for the comparison. The features of the comparison methods are summarized in the Table III.

TABLE I: FEATURE VECTOR LENGTH USED IN EXPERIMENTS

In order to show the the correctness of cluster prominence for the sub-bands, we try to test for all features of GLCM. We evaluated most of them in the directional sub-bands. Here are some classification results of using GLCM texture features. Table II shows the precision rates when using the features of GLCM matrix.

TABLE II: GLCM FEATURES ON WEDGES

\begin{tabular}{lc}
\hline \hline Name of features & Precision $(\%)$ \\
\hline Correlation & 58 \\
Cluster Prominence & 68 \\
Difference entropy & 55 \\
Dissimilarity & 52 \\
Difference variance & 55 \\
Energy & 50 \\
Entropy & 50 \\
Inverse difference & 52 \\
Inverse difference normalized & 56 \\
Information measure of correlation & 58 \\
Maximum probability & 51 \\
Sum entropy & 56 \\
Sum of squares: Variance & 50 \\
Cluster Prominence + Cluster Shade & 63 \\
Cluster Prominence + Cluster Shade+Homogeneity & 52 \\
Correlation+Sum variance+ Sum average & 57 \\
Homogeneity+Correlation+ Sum average & 60 \\
\hline \hline
\end{tabular}

In order to compare the methods, the average classification rates are evaluated for the various parameter sets of each method. Finally, the average rate of each method is computed. The Table III shows these comparisons.

TABLE III: CLASSIFICATION COMPARISON

\begin{tabular}{lcc}
\hline Methods Name & Parameters \& explanation & $\begin{array}{c}\text { Average } \\
\text { Precision }(\%)\end{array}$ \\
\hline GLCM & All statistic values. Using two \\
& pairs $[0,1]$, and $[1,0]$ for \\
& $(\Delta x, \Delta y)$ & 55 \\
Gabor & $2-4$ scales, $4-8$ orientations & 68 \\
SFTA & $4-8$ thresholds & 55 \\
HSCF & 16 Gabors values for low-pass, & 70 \\
& and 2 cluster prominence for \\
& every directional sub-bands (28 & \\
& sub-bands) & \\
\hline \hline
\end{tabular}

\section{CONCLUSIONS}

In this paper, we have presented the novel features for bone texture characterization. The HSCF method is based on contourlet transform and Gabor filter. This is the first result of applying contourlet features for classification of 2D bone 
texture images. For evaluation the performance of the proposed method, we compared HSCF performance against some popular texture such as Haralick, Gabor filters and SFTA algorithm on dataset used in worldwide challenge in bone texture characterization. It is observed that the average precision is higher than that in the related works. Although the HSCF performance is not high enough in practice, but we think that it is the potential direction of using $2 \mathrm{D}$ textures to solve this problem.

\section{ACKNOWLEDGEMENT}

In this paper, we have used dataset from IEEE-ISBI 2014: http://www.univ-orleans.fr/i3mto/challenge-ieee-isbi-bonetexture-characterization. For the non-commercial and research purposes, we hope that they can share and give us the rights to use

\section{REFERENCES}

[1] R. Bartl and B. Frisch, Osteoporosis: Diagnosis, Prevention, Therapy, 2nd ed. Springer, 2009

[2] S. Hough, "Fast and slow bone losers: Relevance to the management of osteoporosis," Drug Aging, vol. 12, pp. 1-7, 1998.
[3] E. Martín-Badosa, A. Elmoutaouakkil, S. Nuzzo, D. Amblard, L. Vico, and F. Peyrin, "A method for the automatic characterization of bone architecture in 3D mice microtomographic images," Comput Med Imaging Graph., vol. 27, pp. 447-458, 2003.

[4] R. M. Haralick, K. Shanmugam, and I. H. Dinstein, "Texture features for image classfication," IEEE Transactions on Systems, Man and Cybernetics, vol. 3, no. 6, pp. 610-621, 1973.

[5] A. F. Costa, G. H. Mamani, and A. J. M. Traina, "An efficient algorithm for fractal analysis of textures," in Proc. 25th SIBGRAPI Conference on Graphics, Patterns and Images (SIBGRAPI), 2012.

[6] B. S. Manjunath and W. Y. Ma, "Texture features for browsing and retrieval of image data," IEEE Transactions on Pattern Analysis and Machine Intelligence, vol. 18, no. 8, pp. 837-842, 1996.

[7] M. N. Do and M. Vetterli, "The contourlet transform: An efficient directional multiresolution image representation," IEEE Transactions on Image Processing, vol. 14, no. 12, pp. 2091-2106, 2005.

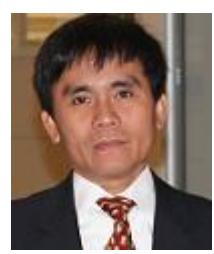

Viet Quoc Ngo received his B.S. degree in information technology from University of Natural Sicence, HCMC in 1989, M.S. degree in computer science in 1997, and $\mathrm{PhD}$ degree in computer science in 2009 from the University of Natural Science, HCMC Vietnam. He is a lecturer in the Faculty of Information Technology at University of Education, HCMC Vietnam. His research interests are image/video processing, computer vision, hybrid algorithms, and biomedical. 